\title{
SOME BASIC PROPERTIES OF THE SURROGATE SUBGRID-SCALE HEAT FLUX IN THE ATMOSPHERIC BOUNDARY LAYER
}

\author{
FERNANDO PORTÉ-AGEL ${ }^{1,3}$, CHARLES MENEVEAU ${ }^{2,3}$ and MARC B. \\ PARLANGE ${ }^{1,3}$ \\ ${ }^{1}$ Department of Geography and Environmental Engineering; ${ }^{2}$ Department of Mechanical \\ Engineering; ${ }^{3}$ Center for Environmental and Applied Fluid Mechanics, The Johns Hopkins \\ University, Baltimore, MD 21218, USA
}

(Received in final form 5 February 1998)

\begin{abstract}
The development of improved subgrid-scale (SGS) models for large-eddy simulation of scalar transport in the atmospheric boundary layer requires an improved understanding of basic properties of the SGS fluxes. High frequency atmospheric wind speed and temperature data sampled at a height of $1.7 \mathrm{~m}$ are used to measure SGS heat fluxes and dissipation of temperature variance, by means of one-dimensional filtering and invoking Taylor's hypothesis. Conditional averaging is used to isolate interesting features of the SGS signals, and to relate them to the large-scale characteristics of the flow, such as the presence of coherent structures. Both mean and conditionally averaged SGS quantities are compared with those obtained using a standard eddy-diffusivity model. Within the limitations imposed by the one-dimensional data analysis, we observe that the model appears unable to reproduce important features of the real signals, such as the negative dissipation of temperature variance associated with strong negative resolved temperature gradients due to the ejection of warm air under unstable atmospheric stability conditions.
\end{abstract}

Keywords: Atmospheric turbulence, Eddy-diffusivity model, Large-eddy simulation, Subgrid-scale heat flux, Subgrid-scale modeling, Turbulent transport

\section{Introduction}

Large-eddy simulation (LES) has become an important tool for the study of transport processes in the atmospheric boundary layer (ABL). It can be used to investigate the unsteady, three dimensional transport of scalar quantities such as temperature, water vapour and pollutants. Since its first applications (e.g., Deardorff, 1970), LES has helped provide a better understanding of the role different factors such as atmospheric stability, surface heterogeneity, and topography, play in land-atmosphere interaction (Nieuwstadt et al., 1991; Shaw and Schumann, 1992; Andrén et al., 1994; Sorbjan, 1996; Dwyer et al., 1997; Albertson and Parlange, 1997).

LES solves the filtered three dimensional unsteady transport equations for momentum and scalar quantities. For instance, consider the governing transport equation for a scalar quantity $\theta$ (e.g., temperature), 


$$
\frac{\partial \theta}{\partial t}+\frac{\partial\left(u_{j} \theta\right)}{\partial x_{j}}=\gamma \frac{\partial^{2} \theta}{\partial x_{i}^{2}}+Q
$$

where $t$ is time, $x_{j}$ is the spatial coordinate in the $j$-direction, $u_{j}$ is the velocity component in the $j$-direction, $\gamma$ is the molecular diffusivity, and $Q$ is a source term. Spatial filtering of the above equation using a filter with characteristic width $\Delta$ yields an equation which is now amenable to numerical solution on a grid with mesh-size of order $\Delta$ :

$$
\frac{\partial \widetilde{\theta}}{\partial t}+\frac{\partial\left(\tilde{u}_{j} \tilde{\theta}\right)}{\partial x_{j}}=-\frac{\partial q_{i}}{\partial x_{i}}+\widetilde{Q}
$$

where the molecular diffusion has been neglected, and the tilde denotes the filtering operation, and $q_{i}$ is the subgrid-scale (SGS) scalar flux in the $i$-direction, defined as:

$$
q_{i}=\widetilde{u_{i} \theta}-\tilde{u_{i}} \tilde{\theta} .
$$

The SGS scalar flux $q_{i}$ represents the contribution of the small scales (smaller than the filter scale) to the total transport and it must be parameterized as a function of the resolved field.

The SGS flux has a significant impact on the dynamics of the filtered scalar variance

$$
\sigma_{\theta}^{2}=\left\langle\frac{1}{2} \widetilde{\theta}^{\prime 2}\right\rangle=\frac{1}{2}\left\langle(\widetilde{\theta}-\langle\widetilde{\theta}|)^{2}\right\rangle
$$

where \langle\rangle denotes ensemble averaging. Since LES produces fluctuating variables, ensemble averaging is important to study the statistics of the resolved fields. Each variable in Equation (2) can be decomposed into its ensemble average plus the deviation from that average (e.g., $\widetilde{\theta}=\langle\widetilde{\theta}\rangle+\widetilde{\theta}^{\prime}$ ). Using this decomposition in Equation (2) and then taking ensemble averages yields the transport equation for the average resolved temperature,

$$
\frac{\partial\langle\widetilde{\theta}\rangle}{\partial t}+\frac{\partial\left(\left\langle\widetilde{u}_{j}\right\rangle\langle\widetilde{\theta}\rangle\right)}{\partial x_{j}}+\frac{\partial\left\langle\widetilde{u}_{j}^{\prime} \widetilde{\theta}^{\prime}\right\rangle}{\partial x_{j}}=-\frac{\partial\left\langle q_{i}\right\rangle}{\partial x_{i}}+\langle\widetilde{Q}\rangle .
$$

Subtraction of Equation (4) from Equation (2) in which all variables are decomposed into their ensemble average and fluctuating parts, and posterior multiplication of all terms by $\widetilde{\theta}^{\prime}$ followed by ensemble averaging, yields the transport equation for $\sigma_{\theta}^{2}$. In the absence of sources and neglecting molecular effects on the resolved scales this is

$$
\frac{\partial \sigma_{\theta}^{2}}{\partial t}+\frac{\partial\left(\left\langle\widetilde{u}_{j}\right\rangle \sigma_{\theta}^{2}\right)}{\partial x_{j}}=-\frac{\partial}{\partial x_{j}}\left(\left\langle\widetilde{u}_{j}^{\prime} \frac{1}{2} \widetilde{\theta}^{\prime 2}\right\rangle+\left\langle q_{i} \widetilde{\theta}^{\prime}\right\rangle\right)-\left\langle\widetilde{u}_{j}^{\prime} \widetilde{\theta}^{\prime}\right\rangle \frac{\partial\langle\widetilde{\theta}\rangle}{\partial x_{j}}-\langle\chi\rangle,
$$


where the first two terms on the right hand side of Equation (5) represent spatial transport due to resolved and unresolved motion, respectively, the third term is production of resolved temperature fluctuations due to mean gradients, and the last term is the ensemble average of

$$
\chi=-q_{j} \frac{\partial \widetilde{\theta}^{\prime}}{\partial x_{j}}
$$

which is the so-called SGS dissipation rate of scalar variance (also equal to the production of unresolved temperature fluctuations). This term is important since it represents the dominant effect of the unresolved motion on $\sigma_{\theta}^{2}$. It typically has an average positive value, indicating a net drain of resolved scalar variance into the subgrid scales.

The formulation of the SGS model in LES has proven to be quite important, especially in the near surface region, where the contribution of the non-resolved field is the largest. Different types of SGS models are currently in use in LES codes for atmospheric flows. For an extensive review, see Mason (1994). These models can be classified as follows:

(1) Eddy-diffusivity models parameterize the SGS fluxes as being proportional to the resolved velocity and temperature gradients. Their simplicity have made them the most popular since they were first introduced by Smagorinsky (1963). The so called dynamic model (Germano et al., 1991; Ghosal et al., 1995) uses information from the resolved field to optimize the value of the free parameter as a function of time and position. Although successfully implemented in a number of engineering flows (see e.g., Akselvoll and Moin, 1996), the application of the dynamic model to atmospheric flows has not yet become common place. Recently, Porté-Agel et al. (1998) have proposed a generalized scale-dependent dynamic procedure, and compared it to the traditional and dynamic Smagorinsky models in LES of the ABL.

(2) Second-order closure models are in theory capable of capturing more details but they require the solution of additional transport equations (Deardorff, 1974), which has precluded their use in LES.

(3) Stochastic backscatter models (Mason and Thomson, 1992; Schumann, 1995) are basically eddy-diffusivity models that introduce random fluctuations in the SGS fluxes in order to account for the transfer (also regarded as backscatter) of scalar variance and kinetic energy from the SGS scales to the resolved field.

The evaluation of the performance of these models has traditionally been based on the comparison between the mean flow properties obtained from the simulation and those measured in the atmosphere. This approach has proven to be somewhat limited in making fundamental progress in SGS modeling because of the large number of additional factors involved whose effects are difficult of disentangle from the SGS model. Such effects include numerical resolution, discretization methods, surface heterogeneity, etc. 
A complementary approach is based on the a priori study of the characteristics and fundamental features of SGS fluxes and their relationship to the large scales of the flow. Such studies are based on well-resolved turbulent fields, from which the SGS fluxes can be computed according to their definition (Equation (3)). This approach was first used with data from Direct Numerical Simulations (DNS) in isotropic turbulence (e.g., Clark et al., 1979; Domaradski et al., 1993), and planar channel flow (e.g., Piomelli et al., 1988; Härtel and Kleiser, 1993) at low Reynolds numbers. Meneveau (1994) and O'Neil and Meneveau (1997) used high frequency hot wire measurements from grid turbulence and in the wake of a cylinder, respectively, to compare the real SGS energy dissipation with the results from several SGS models. Liu et al. (1994) used two-dimensional (2-D) Particle-Image-Velocimetry data obtained in a turbulent jet for similar purposes.

In this study we explore the use of the a priori approach to SGS modeling of atmospheric flows based on field data. Fast response data collected in the surface layer of the $\mathrm{ABL}$ are filtered to compute one-dimensional surrogates for the SGS heat flux, $q_{i}$, and dissipation, $\chi$, of temperature variance. One objective is to document basic features such as their mean values as functions of filter scale $\Delta$. For instance, it is usually argued that at small filter scales, the SGS flux contributes only a small fraction to the total fluxes. However it is important to quantify such statements and explore dependences with $\Delta$. Another objective is to compare the measured SGS quantities with those obtained using a simple eddy-diffusivity model. The modeled heat flux in the $i$-direction is proportional to the derivative of the filtered temperature in that direction

$$
q_{i}^{\bmod }=-\operatorname{Pr}_{T}^{-1} v_{T} \frac{\partial \tilde{\theta}}{\partial x_{i}},
$$

where $\operatorname{Pr}_{T}$ is the so called sub-grid Prandtl number and $v_{T}$ is the turbulent viscosity. $v_{T}$ is derived from the local strain rate and length scale by applying what can be regarded as a three-dimensional version of the mixing-length turbulence model. This leads to a model for $v_{T}$ of the form (Smagorinsky, 1963)

$$
v_{T}=C_{S}^{2} \Delta^{2}|\widetilde{S}|,
$$

where

$$
|\widetilde{S}|=\left(2 \widetilde{S}_{i j} \widetilde{S}_{i j}\right)^{1 / 2}
$$

is the resolved strain-rate magnitude, and

$$
\widetilde{S}_{i j}=\frac{1}{2}\left(\frac{\partial \widetilde{u}_{i}}{\partial x_{j}}+\frac{\partial \tilde{u}_{j}}{\partial x_{i}}\right)
$$

is the resolved strain rate tensor. $C_{S}$ is a non-dimensional parameter which for isotropic, homogeneous turbulence is known to have a value of about 0.17 (Lilly, 
1967). By construction this model gives a scalar flux that is aligned with the scalar gradient. From Equation (6) this means that only positive values of dissipation of scalar variance may occur, and thus the model is regarded as being fully dissipative (the scalar variance at the filter scale can only be dissipated by the subgrid scales).

Another objective of this work is to ascertain the effect of coherent structures on SGS dynamics. In the atmospheric surface layer coherent structures are identifiable in measured scalar (temperature and humidity) time series as "ramps" associated with the sweep-ejection motion of the flow. Ramps are well-defined saw-tooth shaped structures or patterns that occur repeatedly in the scalar signals (e.g., Gao et al., 1989; Shaw et al., 1983, 1989; Gao and Shaw, 1992; Raupach et al., 1991; Katul et al., 1997a). Under diurnal convective (buoyant) conditions, over a relatively hot and wet surface, the coherent structures of the flow are composed of ejection motions transporting warm and moist air upwards, alternating with sweeps of cool and dry air from aloft.

Thus, the comparison between real and modeled SGS quantities includes not only their mean values but also conditional averages, which are intended to evaluate the performance of the model and its capacity to reproduce the local events associated with different parts of the resolved flow.

\section{Experiment}

The atmospheric data used in this study were obtained from a field experiment carried out over a bare soil surface at the Campbell Tract research field of the University of California at Davis during the summer of 1996. Further details of the bare soil site are presented in Parlange et al. (1993) and Albertson et al. (1997).

A three-dimensional sonic anemometer (Gill Instruments/1012R2) placed at a height $(z)$ of $1.70 \mathrm{~m}$ measured the longitudinal $\left(u_{1}\right)$, lateral $\left(u_{2}\right)$ and vertical $\left(u_{3}\right)$ wind velocity components as well as the speed of sound $(c)$ at $18.2 \mathrm{~Hz}$. The absolute air temperature $(\theta)$ was determined from the measured speed of sound using $\theta=\alpha c^{2} / R_{d}$, where $\alpha=C_{p} / C_{v}=1.4, C_{p}$ and $C_{v}$ are the specific heat capacities of dry air under constant pressure and volume, respectively, and $R_{d}$ is the gas constant for dry air. Other meteorological measurements included net radiation, water vapour concentration and the soil surface temperature.

The data presented and analyzed here were obtained over a 60-minute period, from $1607 \mathrm{pm}$ to $1707 \mathrm{pm}$ on June 25, 1996. Table I has a summary of the mean meteorological and turbulence conditions. No significant change in these conditions was observed throughout the measurement period. From Table I, the stability parameter $z / L$ was -0.039 , indicating unstable atmospheric stability conditions, where $L$ is the Obukhov length,

$$
L=\frac{-u_{*}^{3}}{\kappa g H /\left(\rho C_{p} T\right)},
$$


TABLE I

Summary of meteorological and turbulence conditions during the experiment.

\begin{tabular}{ll}
\hline Meteorological and turbulence conditions \\
\hline Mean horizontal wind speed $\left(\left\langle u_{1}\right\rangle\right)$ & $6.41 \mathrm{~m} \mathrm{~s}^{-1}$ \\
Mean air temperature $(\langle\theta\rangle)$ & $23.11^{\circ} \mathrm{C}$ \\
Sensible heat flux $(H)$ & $182.9 \mathrm{~W} \mathrm{~m}^{-2}$ \\
Latent heat flux $(L E)$ & $12.2 \mathrm{~W} \mathrm{~m}^{-2}$ \\
RMS temperature $\left(\sigma_{T}\right)$ & $0.82^{\circ} \mathrm{C}$ \\
RMS vertical velocity $\left(\sigma_{u_{3}}\right)$ & $0.47 \mathrm{~m} \mathrm{~s}^{-1}$ \\
RMS horizontal velocity $\left(\sigma_{u_{1}}\right)$ & $1.15 \mathrm{~m} \mathrm{~s}^{-1}$ \\
Friction velocity $\left(u_{*}\right)$ & $0.43 \mathrm{~m} \mathrm{~s}^{-1}$ \\
Momentum roughness length $\left(z_{o}\right)$ & $0.004 \mathrm{~m}^{-}$ \\
Obukhov length $(L)$ & $-43.78 \mathrm{~m}$ \\
\hline
\end{tabular}

$u_{*}\left(=[\tau / \rho]^{1 / 2}=\left[-\left\langle u_{1} u_{3}\right\rangle\right]^{1 / 2}\right)$ is the friction velocity, $\tau$ is the surface shear stress, $\rho$ is the density of air, $H$ is the sensible heat flux, $T$ is the mean air temperature, $g$ is the gravitational acceleration, and $\kappa(=0.4)$ is the von Karman constant.

The power spectra for the streamwise velocity and temperature signals are computed and presented in Figure 1. They are obtained from 30 segments of 1024 points each, using a Barlett window (Press et al., 1992). In Figure 1 the power spectrum $\left(E_{k}\right.$ for a generic signal $k$ ) is normalized as $E_{k} / \sigma_{k}^{2} z$, where $\sigma_{k}^{2}$ is the variance of the signal. The wavenumber $k_{1}$ is based on the sampling frequency, using Taylor's hypothesis, and it is normalized by $z$. The slope of the spectrum is $-5 / 3$ for wavenumbers $k_{1}>0.7 \mathrm{~m}^{-1}$, indicative of the inertial subrange. At smaller wavenumbers, the slope is lower.

\section{Data Analysis}

\subsection{SGS HEAT FLUX AND DISSIPATION OF TEMPERATURE VARIANCE}

The streamwise SGS heat flux, $q_{1}$, is computed according to its definition (Equation (3)) $q_{1}=\widetilde{u_{1} \theta}-\widetilde{u}_{1} \widetilde{\theta}$. Since the measurements were taken at one point, three dimensional filtering is not feasible. Instead, the filtering is performed in time and is interpreted as one-dimensional spatial filtering in the streamwise direction by means of Taylor's frozen flow hypothesis. Taylor's hypothesis in the inertial subrange is often applied (e.g., Peltier et al., 1996; Kiely et al., 1996; Katul et al., 1997b), and was justified by Wyngaard and Clifford (1977). 


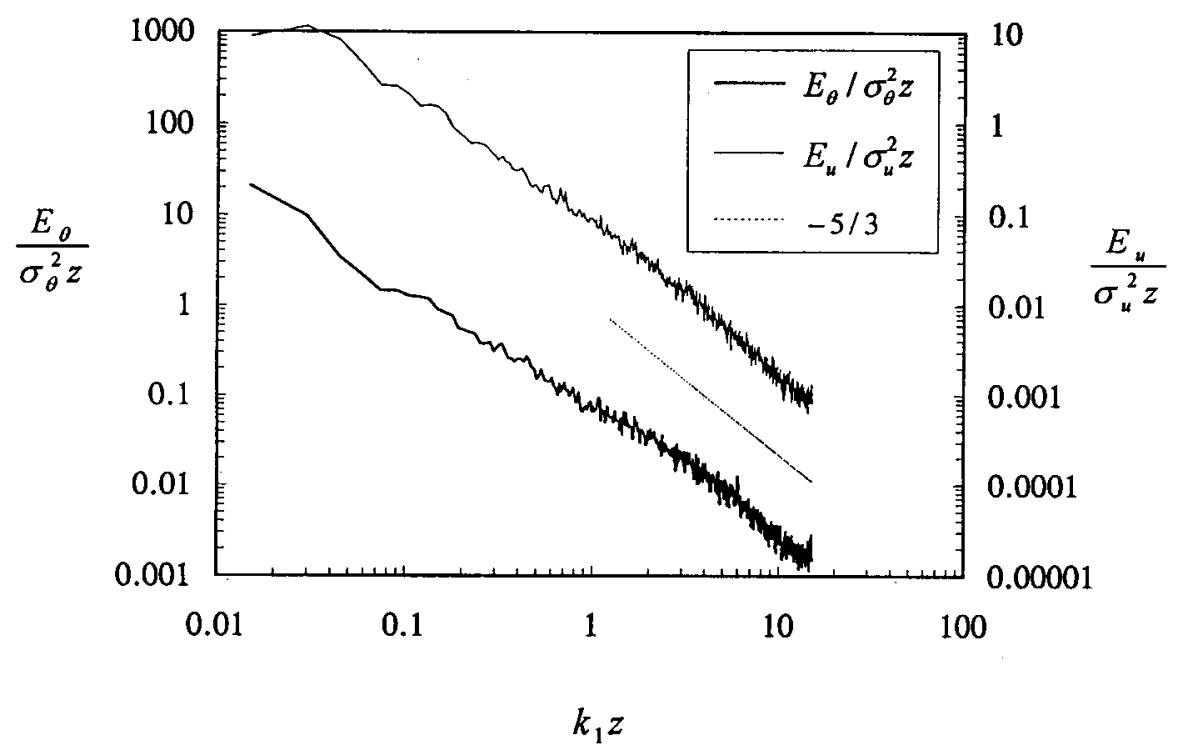

Figure 1. Normalized power spectra for the streamwise velocity and the temperature signals. The $-5 / 3$ power law is also shown.

For a generic variable $y(x)$, the filtered variable $\tilde{y}(x)$ is computed by convolution with a filter function $G_{\Delta}: \tilde{y}(x)=\int y(x) G_{\Delta}\left(x-x^{\prime}\right) \mathrm{d} x^{\prime}$, where $x$ is the streamwise spatial coordinate, and $x^{\prime}$ is an integration variable. For computational convenience the filtering is done in wave space using the Fast Fourier Transform (Press et al., 1992). The results presented here were obtained using a Gaussian filter whose Fourier transform is of the form:

$$
\widehat{G}_{\Delta}\left(k_{1}\right)=\exp \left(-\frac{k_{1}^{2} \Delta^{2}}{24}\right)
$$

where $k_{1}$ is the wave number and $\Delta$ the filter size. Two other filter types, spectral cutoff and tophat, were also tested and similar results were obtained. This is in agreement with previous research (e.g., Meneveau, 1994) which found that the impact of using different filter types on the SGS flux, SGS dissipation, model coefficients, etc. was small.

The SGS heat flux modeled according to the Smagorinsky eddy-diffusivity model, Equations (7) and (8), for the streamwise direction is

$$
q_{1}^{\bmod }=-\operatorname{Pr}_{T}^{-1} C_{S}^{2} \Delta^{2}|\widetilde{S}| \frac{\partial \widetilde{\theta}}{\partial x_{1}}
$$




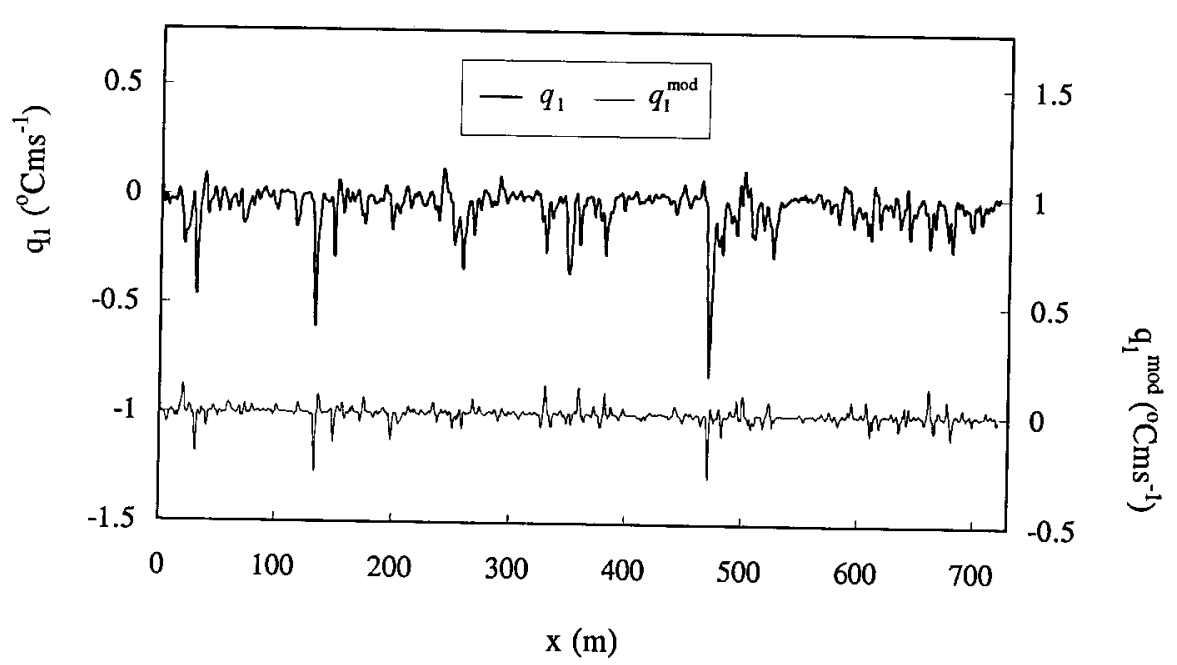

Figure 2. Portion of the real and modeled SGS heat flux signals, evaluated from data obtained in the atmospheric boundary layer, using 1-D filtering and Taylor's hypothesis.

The resolved strain rate $|\widetilde{S}|$ is computed using a one-dimensional approach. By assuming that the smallest scales of the resolved motion are isotropic, the following equality holds:

$$
\left\langle\widetilde{S}_{i j} \widetilde{S}_{i j}\right\rangle=\frac{15}{2}\left\langle\widetilde{S}_{11}^{2}\right\rangle
$$

(see Monin and Yaglom, 1971). This allows the average resolved strain rate to be computed using only the 1-1 component of the strain rate tensor. We will employ this assumption for the instantaneous fields, and write

$$
|\widetilde{S}|=\left(2 \widetilde{S}_{i j} \widetilde{S}_{i j}\right)^{1 / 2} \approx \sqrt{15}\left|\frac{\partial \widetilde{u}_{1}}{\partial x_{1}}\right| .
$$

The spatial derivatives are computed from the time derivatives invoking Taylor's frozen flow hypothesis. The coefficient $\operatorname{Pr}_{T}^{-1} C_{S}^{2}$ is set based on SGS dissipation arguments (see below).

In Figure 2 a segment of the real and modeled SGS heat fluxes is presented. There is a general coincidence of regions in which the fluctuations in the signals are stronger. However the model clearly underestimates the magnitude of these fluctuations.

The streamwise term of the SGS dissipation of the temperature variance is computed according to its definition $\chi_{1}=-q_{1} \partial \widetilde{\theta}^{\prime} / \partial x_{1}$. Similarly, the modeled SGS dissipation is computed with the modeled SGS flux $\chi_{1}^{\bmod }=-q_{1}^{\bmod } \partial \widetilde{\theta}^{\prime} / \partial x_{1}$. Due to the 1-D filtering and the fact that only a single term (out of three) is available, the SGS quantities will be termed "one-dimensional surrogates". 


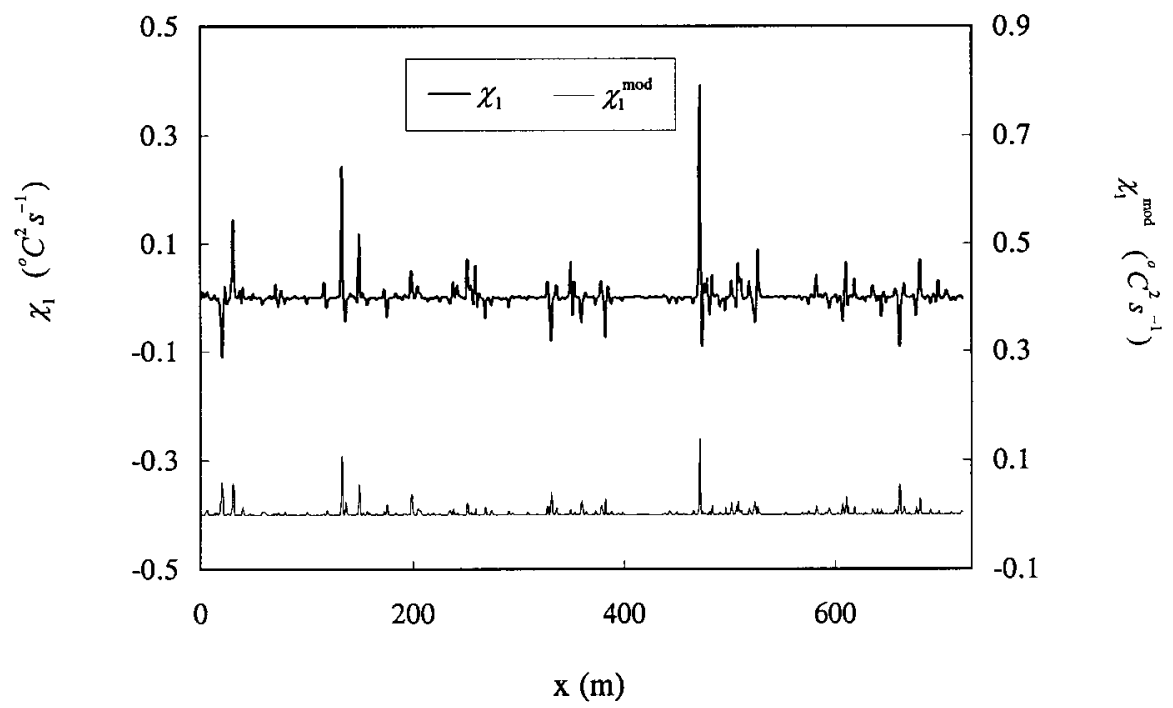

Figure 3. Portion of the real and modeled 1-D surrogate of SGS dissipation of temperature variance.

In Figure 3 a segment of the real and modeled 1-D surrogate of SGS dissipation of resolved temperature variance is presented. The eddy-diffusivity model is by construction fully dissipative, i.e., the scalar variance at the filter scale can only be dissipated by the subgrid scales. Hence, the model is unable to reproduce negative values of the SGS dissipation, which do occur in the real surrogate signal due to backscatter of temperature variance (see Figure 3 ).

The product in Equation (10) $\operatorname{Pr}_{T}^{-1} C_{S}^{2}$ is determined, or calibrated, by forcing the Smagorinsky model to reproduce the correct mean SGS dissipation of temperature variance, i.e., we evaluate

$$
\operatorname{Pr}_{T}^{-1} C_{S}^{2}=\frac{-\left\langle q_{1} \partial \tilde{\theta} / \partial x_{1}\right\rangle}{\left\langle\Delta^{2}|\widetilde{S}|\left(\partial \widetilde{\theta} / \partial x_{1}\right)^{2}\right\rangle}
$$

at some scale (we choose $\Delta=4.27 \mathrm{~m}$ ).

Murray et al. (1996) used data from a DNS of fully developed turbulent channel flow to show that temporal filtering of a single-point data gives similar results to one-dimensional filtering in the streamwise direction. They also showed that onedimensional filtering gave similar results to three-dimensional filtering above 50 wall units from the bottom of the channel, where the turbulent eddies tend towards isotropy. Considering that in this experiment the ratio between the height of the instrument $(z)$ and the roughness length $\left(z_{o}\right)$ is 425 , the sampling point is high enough to be away from the strongly anisotropic near-wall region. However, since the filtering will be done at scales near and above the measurement height $z$, largescale anisotropy could affect the results. 


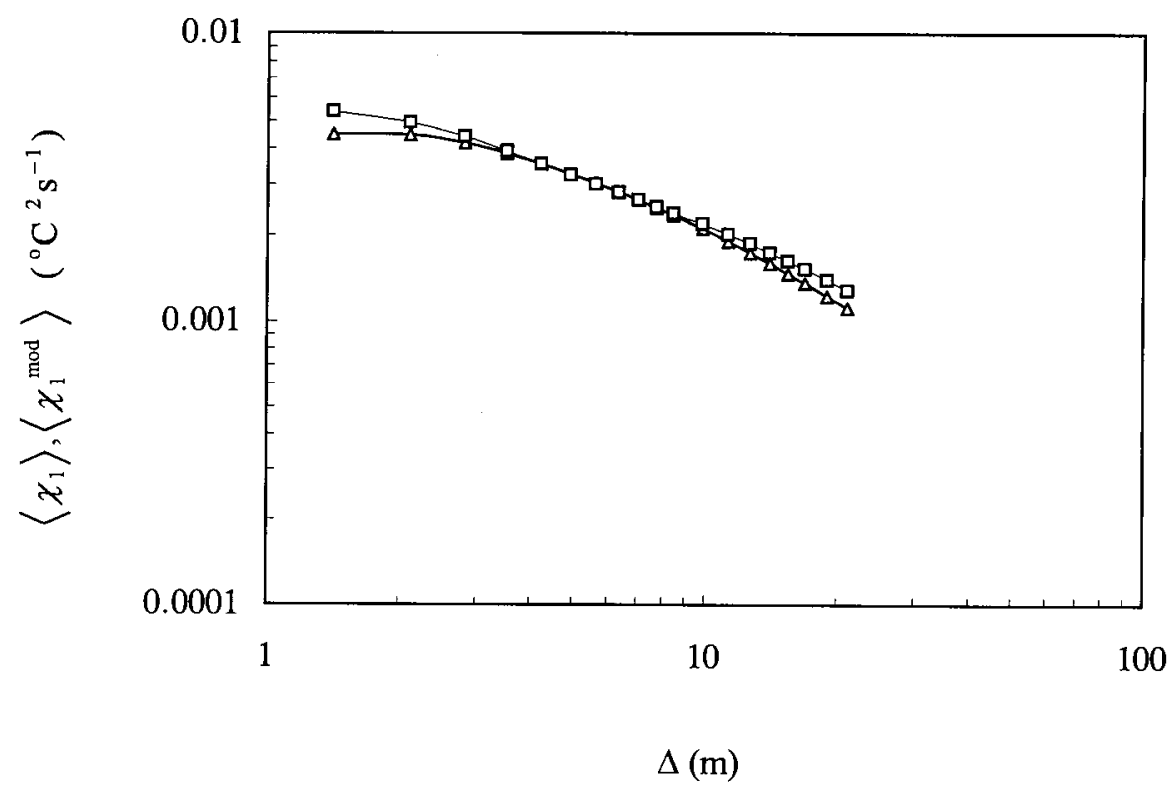

Figure 4. Mean surrogate SGS dissipation of temperature variance $\left\langle\chi_{1}\right\rangle$ (triangles and dark line) as function of filter width $\Delta$. Also shown is the mean modeled SGS dissipation $\left\langle\chi_{1}^{\text {mod }}\right\rangle$ (squares and light line).

\subsection{INFLUENCE OF FILTER WIDTH}

In Figure 4 the influence of the filter width $\Delta$ on the mean real and modeled 1-D surrogates of SGS dissipation of temperature variance is presented. The product $\operatorname{Pr}_{T}^{-1} C_{S}^{2}$ is set to 0.0143 to allow the model to reproduce the mean real dissipation of temperature variance at scale $\Delta=4.27 \mathrm{~m}$. Since it has been found, based on 1-D filtering, that $C_{S} \sim 0.06$ (Meneveau, 1994; O'Neil and Meneveau, 1997), the estimated value of the subgrid Prandtl number is 0.25 . This value is lower than commonly reported values $\left(\operatorname{Pr}_{T} \sim 0.4\right)$, possibly due to effects of 1-D filtering. Interestingly, we observe from Figure 4 that the same value of $\operatorname{Pr}_{T}^{-1} C_{S}^{2}$ is able to reproduce the mean dissipation of the temperature variance for a range of filter widths.

In Figure 5 the influence of the filter width $\Delta$ on the mean measured and modeled SGS fluxes is examined. Note that in Figure 5, for presentation purposes, the fluxes have been multiplied by -1 . In double logarithmic scale, the slope of the real SGS flux curve decreases from a value of about 1.2 for $\Delta=1.7 \mathrm{~m}$ to a value of 0.55 for $\Delta=20.0 \mathrm{~m}$. In the inertial range, heuristic scaling arguments suggest that the flux scales as $\Delta^{2 / 3}$, since it is (dimensionally) given by the product of a velocity and scalar difference, each contributing $\Delta^{1 / 3}$. The anisotropy of the flow and the proximity of the measurement point to the ground, relative to the magnitude of $\Delta$, seem to determine the departure from a theoretical $2 / 3$ slope. Note that for all filter 


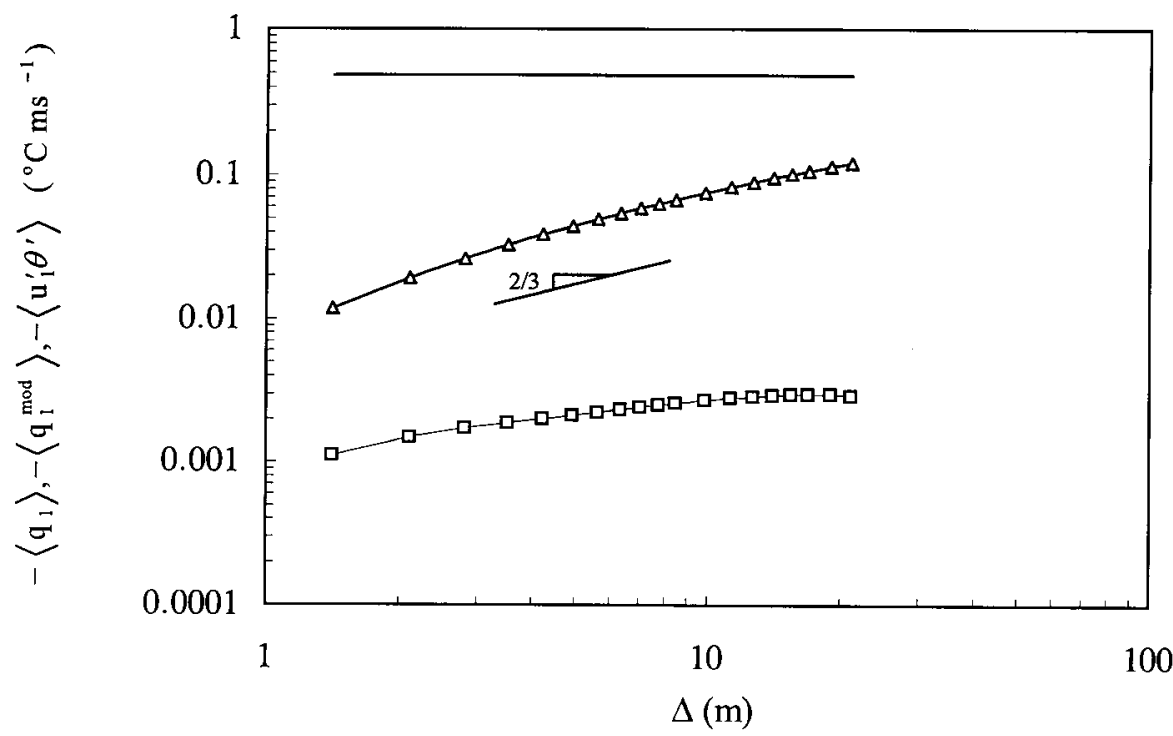

Figure 5. Mean real SGS heat flux $\left\langle q_{1}\right\rangle$ (triangles and dark line) as function of filter width $\Delta$. Also shown is the mean modeled SGS heat flux $\left\langle q_{1}^{\text {mod }}\right\rangle$ (squares and light line). The top line corresponds to the traditional total turbulent heat flux $\left\langle u_{1}^{\prime} \theta^{\prime}\right\rangle$. The $2 / 3$ slope is also shown. Note that for presentation purposes all the fluxes have been multiplied by -1 .

widths considered the model underpredicts the mean SGS flux by a factor of 10 and more. The model also underpredicts the slope of the curve.

\subsection{Conditionally AVERAGED QUANTITIES}

As can be seen in Figures 2 and 3 the SGS flux and dissipation series have a high degree of intermittency. In order to isolate important features of such highly intermittent signals, we perform conditional averaging. We define the conditional average of the signal $Y(x)$ under the condition $C$ in a window of size $W$ according to

$$
\begin{aligned}
& \langle Y(x) \mid C\rangle\left(x^{\prime}\right)=\frac{1}{n} \sum_{i=1}^{n}\left(x_{i}+x^{\prime}\right) \text { with } \\
& -\frac{W}{2} \leq x^{\prime} \leq+\frac{W}{2},
\end{aligned}
$$

where $x_{i}$ (with $1<i<n$ ) are the points where $Y\left(x_{i}\right)$ satisfies the condition $C$. From here on $x$ (instead of $x_{1}$ ) denotes the streamwise coordinate.

Both the SGS flux and the SGS dissipation are conditionally averaged. In order to isolate the different parts of the temperature fields, which may be indicative of 
different regions within coherent structures of the flow, the conditions used are based on the derivative of the filtered resolved temperature $\partial \widetilde{\theta} / \partial x$. Three conditions are used.

Condition I: the derivative being larger than its root mean square value $(\partial \widetilde{\theta} / \partial x>$ $\left.\sigma_{\partial \tilde{\theta} / \partial x}\right)$, which using Taylor's hypothesis corresponds to a temperature drop in the time series. This condition tends to highlight the decreasing part of the ramp structures (saw-tooth shaped structures in the signal). Condition II: $\partial \widetilde{\theta} / \partial x<-\sigma_{\partial \widetilde{\theta} / \partial x}$, which corresponds to a temperature increase in the time series, tending to highlight the rising part of the ramp structures. Condition III: $-\sigma_{\partial \widetilde{\theta} / \partial x}<\partial \widetilde{\theta} / \partial x<\sigma_{\partial \widetilde{\theta} / \partial x}$ corresponding to small (positive and negative) temperature gradients. The selection of the conditioning threshold, $\sigma_{\partial \tilde{\theta} / \partial x}$, is arbitrary and is selected simply to guarantee that it is large enough to isolate the local effects, and small enough to ensure the convergence of conditional averages. The width of the averaging window, $W$, was set to 9.5 times the filter width. This has no effect on the value of the average, but guarantees a complete description since the conditional average asymptotes to the overall average as we move away from the center of the averaging window (at $x^{\prime}=0$ ). The conditional averages presented below correspond to a filter width of $\Delta=4.27 \mathrm{~m}$ and an averaging window of $W=40.5 \mathrm{~m}$.

Figure 6 shows the conditional averages for the real and modeled SGS heat fluxes, under Condition I $\left(\partial \widetilde{\theta} / \partial x>\sigma_{\partial \widetilde{\theta} / \partial x}\right)$. The result for the mean heat flux at the center of the averaging window (i.e., $x^{\prime}=0$ ) indicates that a relatively large negative flux is associated with a strong drop in the temperature signal. The negative sign of the heat flux can be explained by considering that the temperature drop is associated with a local increase in the streamwise velocity of the flow. This is straightforward to understand considering the negative correlation between the streamwise and vertical velocity fluctuations $\left(u_{1}^{\prime}\right.$ and $\left.u_{3}^{\prime}\right)$. A localized increase in $u_{1}$ $\left(u_{1}^{\prime}>0\right)$ is associated with a decrease in $u_{3}\left(u_{3}^{\prime}<0\right)$ i.e., a sweep of air from aloft. Since the air aloft is relatively cooler than the air below, this explains the reduction in temperature and thus the negative streamwise heat flux. The eddy-diffusivity model, although able to reproduce the same qualitative trend, underpredicts the magnitude of the flux near $x^{\prime}=0$. Recall that the coefficients have been calibrated to ensure agreement of the overall average dissipation (i.e., at $\left|x^{\prime}\right| \rightarrow \infty$ ).

The conditional averages for the real and modeled 1-D surrogates of SGS dissipation of temperature variance under Condition I are presented in Figure 7. A larger than average positive dissipation occurs when there is a negative temperature gradient. This indicates that there is a larger amount of temperature variance being transferred from the large (resolved) to the small (subgrid) scales of the flow. Again, the eddy-diffusivity model tends to follow the same trend, but clearly underpredicts the value of the dissipation.

The good convergence of the conditional averages for the real and modeled SGS heat fluxes and 1-D surrogates of dissipation, under Condition I, is illustrated in Figure 8, where the conditional averages at the center point of the averaging 


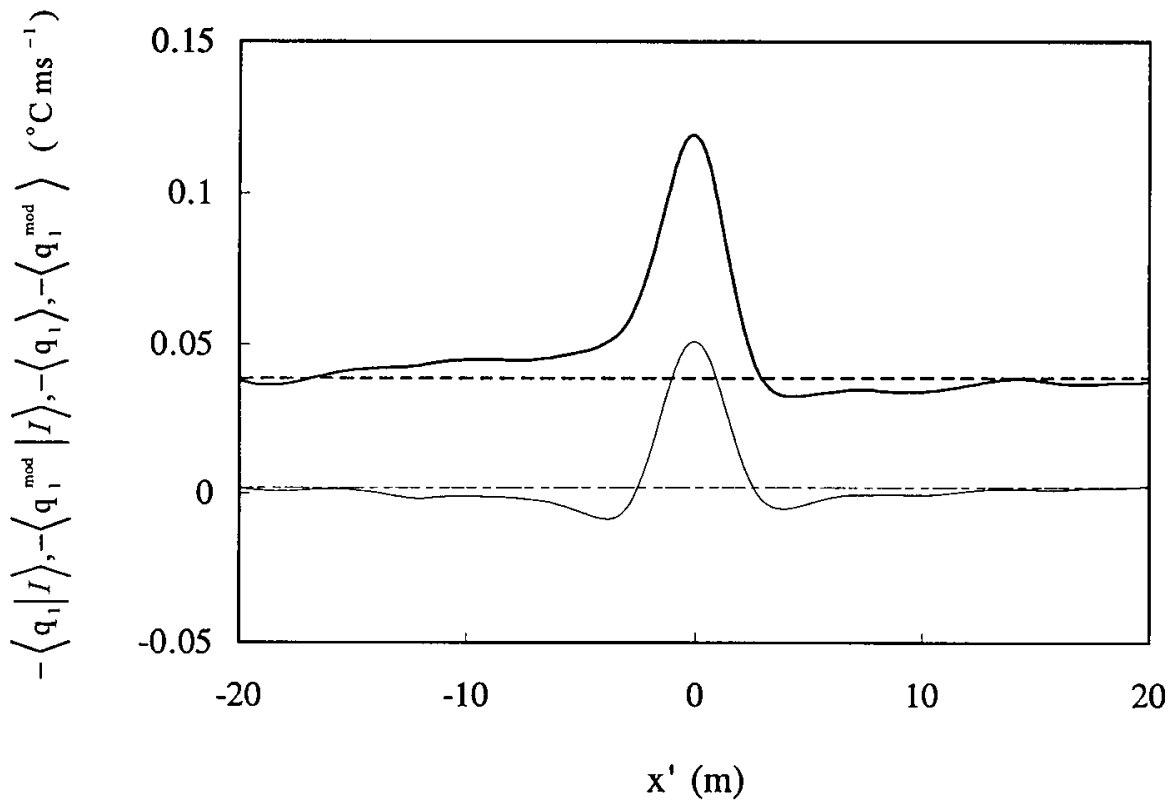

Figure 6. Mean and conditionally averaged surrogate SGS heat flux, for Condition I $\left(\partial \widetilde{\theta} / \partial x>\sigma_{\partial \widetilde{\theta} / \partial x}\right)$. Globally averaged real flux $\left\langle q_{1}\right\rangle$ : dark dashed line; conditionally averaged real flux $\left\langle q_{1} \mid \mathrm{I}\right\rangle$ : dark line; globally averaged modeled flux $\left\langle q_{1}^{\text {mod }}\right\rangle$ : light dashed line; conditionally averaged modeled flux $\left.\left\langle q_{1}^{\bmod }\right| \mathrm{I}\right)$ : light line. Note that for presentation purposes all the fluxes have been multiplied by -1 .

window $\left(x^{\prime}=0\right)$ are plotted against the number of points $(n)$ that are used to compute the conditional average, according to Equation (11).

The conditional averages for the real and modeled SGS heat fluxes, under Condition II $\left(\partial \widetilde{\theta} / \partial x<-\sigma_{\partial \widetilde{\theta} / \partial x}\right)$ are given in Figure 9. In this case the real flux does not depart much from its average value. The negative sign of the flux can be explained by considering that the warming seen at the measurement height is associated with a local decrease in $u_{1}$ which, due to the negative correlation between the velocity fluctuations $\left\langle u_{1}^{\prime} u_{3}^{\prime}\right\rangle$, is associated with an ejection of hot air from below.

In Figure 10 the conditional averages for the real and modeled 1-D surrogates of SGS dissipation of temperature variance under Condition II are plotted. The real signal displays a negative dissipation which indicates that there is backscatter of temperature variance, i.e., temperature variance being transferred from the subgrid scales of the flow to the resolved field. The eddy-diffusivity model fails to reproduce that backscatter because it is fully dissipative by construction.

The influence of the filter width $\Delta$ on the mean and conditionally averaged surrogate of the SGS dissipation of temperature variance under the three conditions (I, II, III) is displayed in Figure 11. These values correspond to the conditional averages at the center points of the averaging window $\left(x^{\prime}=0\right)$. As pointed out 


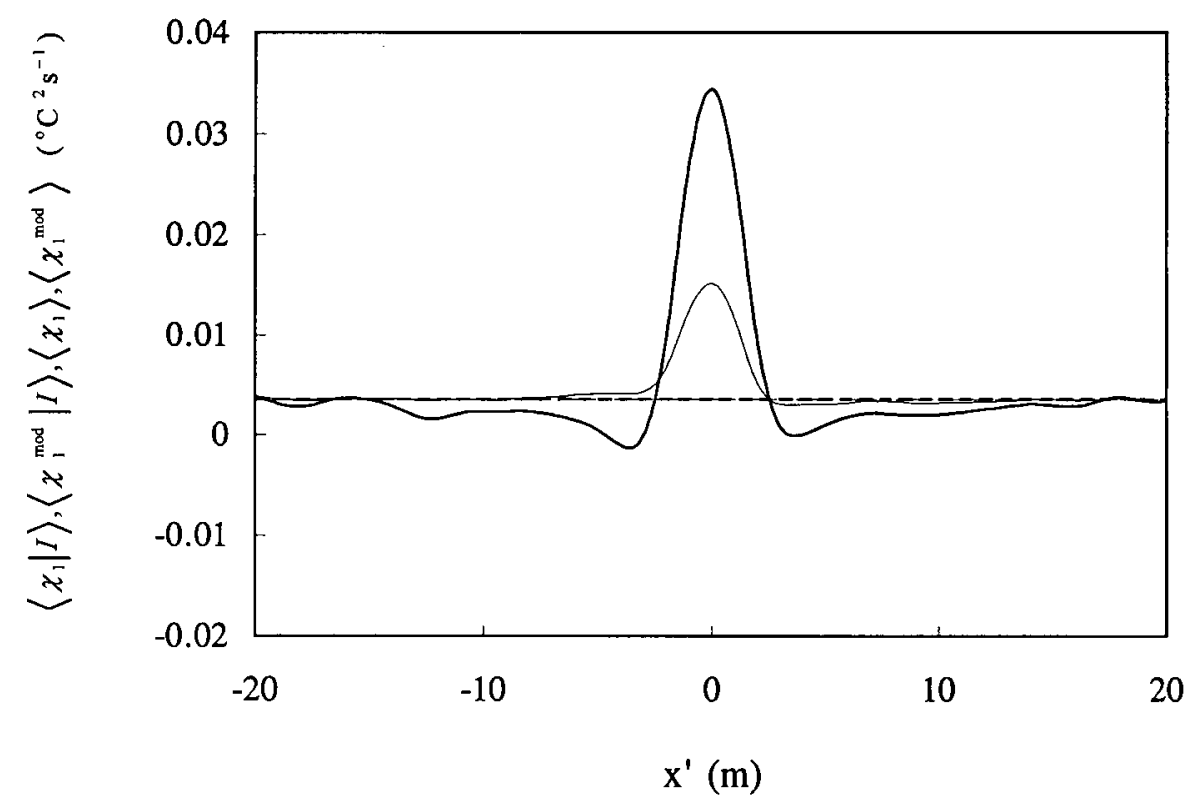

Figure 7. Mean and conditionally averaged surrogate SGS dissipation of temperature variance, for Condition I $\left(\partial \widetilde{\theta} / \partial x>\sigma_{\partial \tilde{\theta} / \partial x}\right)$. Mean real dissipation $\left\langle\chi_{1}\right\rangle$ : dark dashed line; conditionally averaged real dissipation $\left\langle\chi_{1} \mid \mathrm{I}\right\rangle$ : dark line; mean modeled dissipation $\left\langle\chi_{1}^{\text {mod }}\right\rangle$ : light dashed line (agreeing with the real value by construction); conditionally averaged modeled dissipation $\left\langle\chi_{1}^{\bmod } \mid \mathrm{I}\right\rangle$ : light line.

above, Condition I, associated with sweeps of cool air, has the largest individual (per event) contribution with relatively large positive values to the net dissipation of scalar variance. Condition II, associated with ejections of warm air has the opposite effect, with a negative net dissipation, indicating a transfer of scalar variance from the subgrid scales to the resolved field. Condition III, associated with small (positive and negative) temperature gradients, appears to have no net effect. The increment in the filter width $\Delta$ has the effect of reducing the magnitude of all the averages in approximately the same proportion.

In Figure 12 the influence of the filter width $\Delta$ on the mean and conditionally averaged SGS heat flux under the three conditions (I, II, and III) is presented. Sweep events associated with Condition I have the largest individual contribution to the mean SGS heat flux, with relatively large negative values. Ejection events associated with Condition II have a smaller negative contribution to the overall average. As opposed to the surrogate of the SGS dissipation of the temperature variance, Condition III, associated with small (positive or negative) temperature gradients, has a negative net contribution to the overall SGS heat flux, although smaller in magnitude than those from the other events. The increment in the filter width $\Delta$ has the effect of increasing the magnitude of all the averages in approximately the same proportion. 


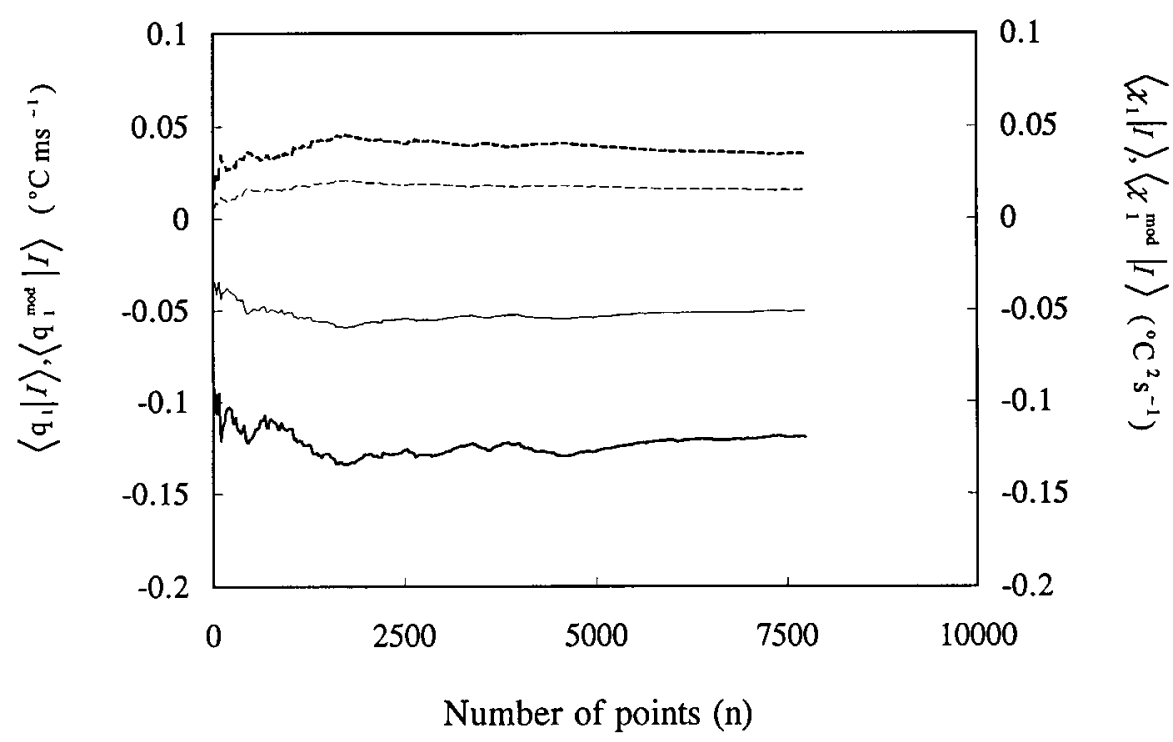

Figure 8. Running conditional averages at $x^{\prime}=0$, under Condition I $\left(\partial \widetilde{\theta} / \partial x>\sigma_{\partial \widetilde{\theta} / \partial x}\right)$, as a function of the number of points $(n)$ used for the averaging. Real flux $\left\langle q_{1} \mid \mathrm{I}\right\rangle$ : dark line; modeled flux $\left\langle q_{1}^{\bmod } \mid \mathrm{I}\right\rangle$ : light line; real dissipation $\left\langle\chi_{1} \mid \mathrm{I}\right\rangle$ : dark dashed line; modeled dissipation $\left\langle\chi_{1}^{\bmod } \mid \mathrm{I}\right\rangle$ : light dashed line.

\section{Conclusions}

SGS heat flux and dissipation of temperature variance have been computed from high resolution data collected at $1.70 \mathrm{~m}$ above a uniform flat bare soil under unstable conditions, by means of one-dimensional filtering, invoking Taylor's hypothesis.

Conditional averaging aided in relating the magnitude of the SGS quantities to sweep and ejection characteristics of the flow. Sweeps of cool air from aloft, associated with high positive temperature gradients, have the largest impact, with a negative SGS heat flux and a positive SGS dissipation of temperature variance. Ejections of warm air from below, associated with high negative temperature gradients, have a relatively smaller contribution with an also negative heat flux, but with a negative SGS dissipation, indicating transfer of temperature variance from the subgrid scales to the resolved field. Components of the flow structures associated with small gradients of temperature have relatively little individual effect. They result in a negative heat flux and negligible dissipation.

Mean and conditionally averaged SGS quantities computed using an eddy-diffusivity model show poor agreement with the real values. By adjusting the parameter $\operatorname{Pr}_{T}^{-1} C_{S}^{2}$, the model reproduces the mean SGS dissipation rate. However, the model underpredicts the mean SGS heat flux by a factor of 10 or more. Although able to reproduce the qualitative trend of the SGS quantities associ- 


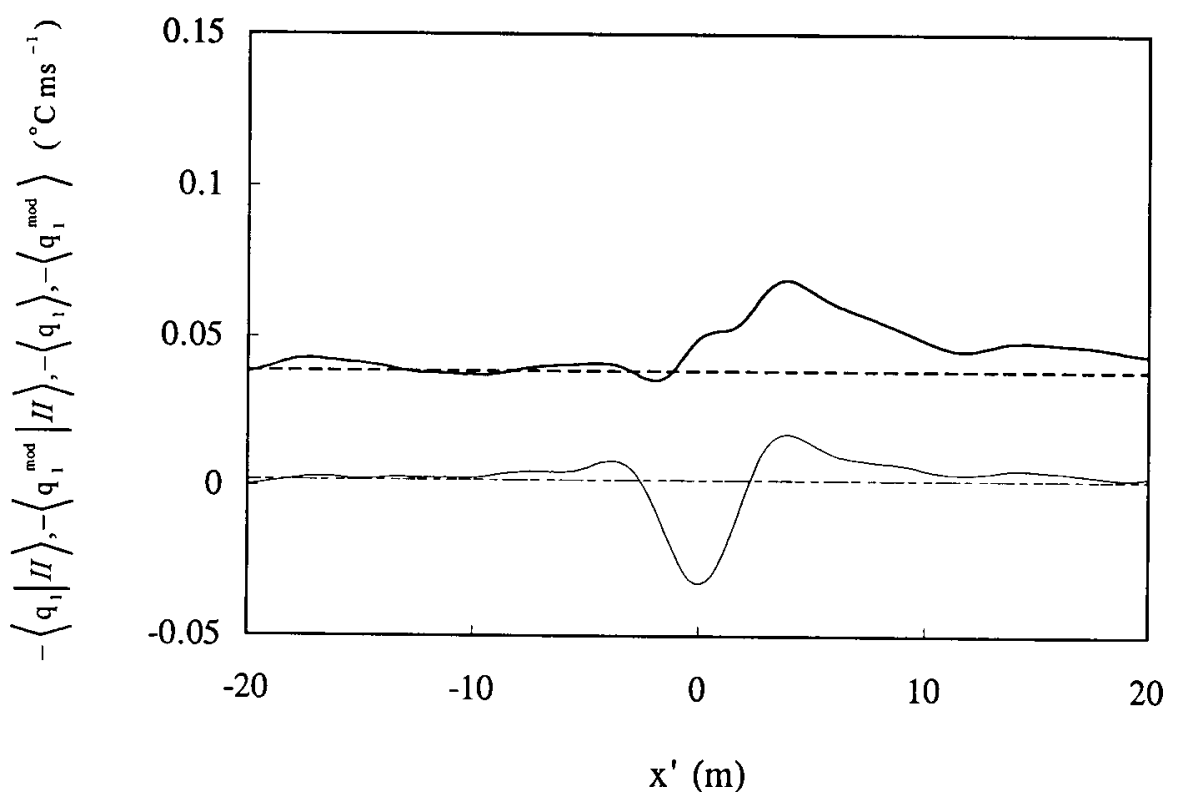

Figure 9. Mean and conditionally averaged surrogate SGS heat flux, for Condition II $(\partial \tilde{\theta} / \partial x<$ $\left.-\sigma_{\partial \tilde{\theta} / \partial x}\right)$. Globally averaged real flux $\left\langle q_{1}\right\rangle$ : dark dashed line; conditionally averaged real flux $\left\langle q_{1} \mid \mathrm{II}\right\rangle$ : dark line; globally averaged modeled flux $\left\langle q_{1}^{\text {mod }}\right\rangle$ : light dashed line; conditionally averaged modeled flux $\left\langle q_{1}^{\bmod } \mid \mathrm{II}\right\rangle$ : light line. Note that for presentation purposes all the fluxes have been multiplied by -1 .

ated with sweep events, the model clearly fails in the ejection events. This is due to the fact that the model is by construction fully dissipative, and therefore unable to reproduce counter-gradient fluxes, i.e., negative SGS dissipation (backscatter) of temperature variance associated with ejections of warm air under unstable conditions.

The results presented in this paper show the potential of using conditional averaging to provide information about the characteristics of the real and modeled SGS quantities associated with distinct events of the resolved field, such as coherent structures. This study constitutes an initial step in the use of the a priori approach to study SGS modeling for atmospheric flows. Further research should extend this type of analysis to other existing SGS models (e.g., dynamic, stochastic backscatter) using data obtained under different stability and surface roughness conditions. The assumptions and simplifications associated with the use of one-dimensional analysis (Taylor's hypothesis, 1-D filtering, and 1-D surrogates) may influence the quantitative details of the results. However, trends and qualitative results are not likely to be significantly affected. Clearly, more extensive data should be used to reduce the need for lower-dimensional surrogate variables. For example, 2-D particle image velocimetry has been applied successfully in the past (Liu et al., 


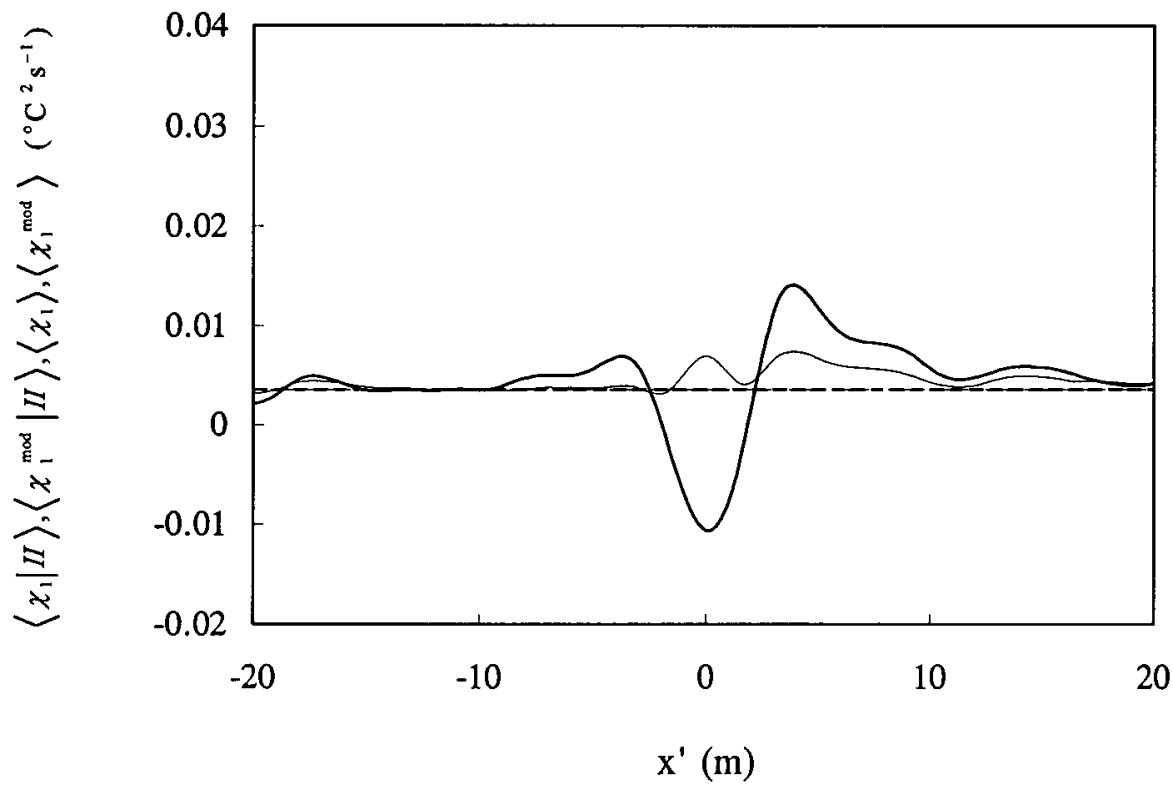

Figure 10. Mean and conditionally averaged surrogate SGS dissipation of temperature variance, for Condition II $\left(\partial \tilde{\theta} / \partial x<-\sigma_{\partial \tilde{\theta} / \partial x}\right)$. Mean real dissipation $\left\langle\chi_{1}\right\rangle$ : dark dashed line; conditionally averaged real dissipation $\left\langle\chi_{1} \mid \mathrm{II}\right\rangle$ : dark line; mean modeled dissipation $\left\langle\chi_{1}^{\text {mod }}\right\rangle$ : light dashed line (agreeing with the real value by construction); conditionally averaged modeled dissipation $\left\langle\chi_{1}^{\bmod } \mid \mathrm{II}\right\rangle$ : light line.

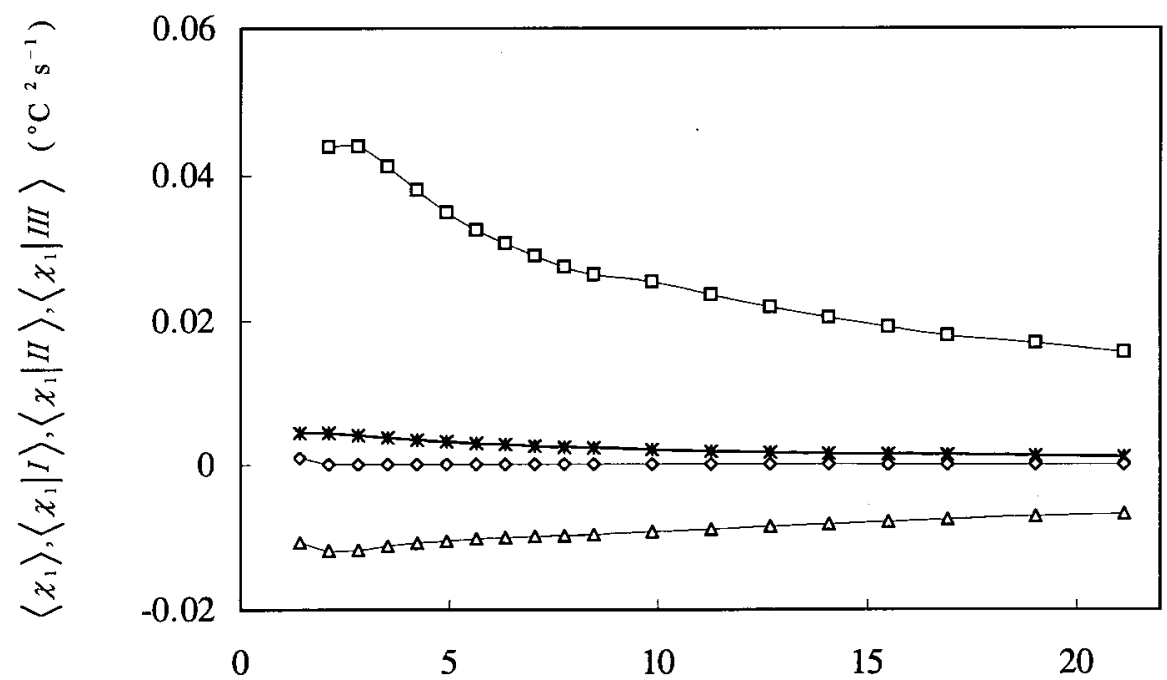

$\Delta(\mathrm{m})$

Figure 11. Effect of filter width $\Delta$ on mean and conditionally averaged surrogate SGS dissipation at $x^{\prime}=0 .\left\langle\chi_{1}\right\rangle$ : stars and dark line; $\left\langle\chi_{1} \mid \mathrm{I}\right\rangle$ : squares and light line; $\left\langle\chi_{1} \mid \mathrm{II}\right\rangle$ : triangles and light line; $\left\langle\chi_{1} \mid \mathrm{III}\right\rangle$ : diamonds and light line. 


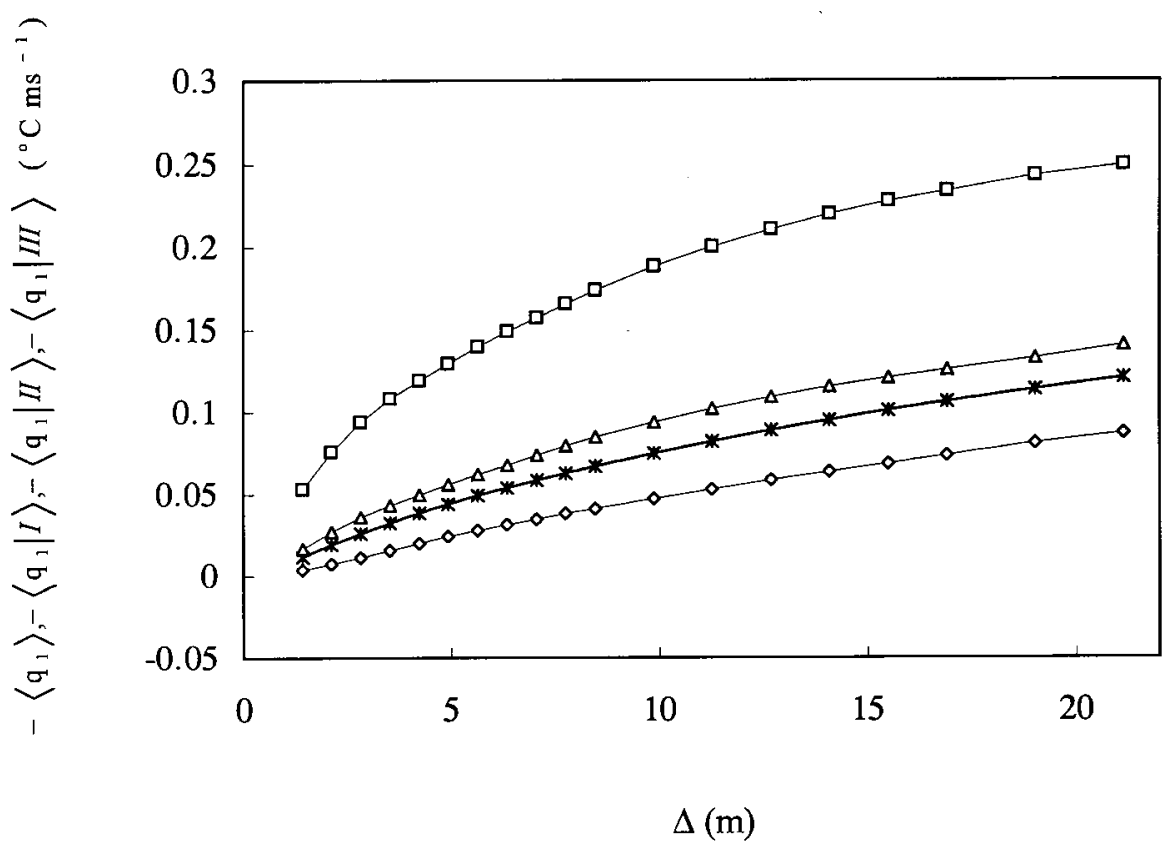

Figure 12. Effect of filter width $\Delta$ on mean and conditionally averaged surrogate SGS heat fluxes at $x^{\prime}=0 .\left\langle q_{1}\right\rangle$ : stars and dark line; $\left\langle q_{1} \mid \mathrm{I}\right\rangle$ : squares and light line; $\left\langle q_{1} \mid \mathrm{II}\right\rangle$ : triangles and light line; $\left\langle q_{1} \mid \mathrm{III}\right\rangle$ : diamonds and light line. Note that for presentation purposes the fluxes have been multiplied by -1 .

1994). For atmospheric flows, LIDAR-based velocimetry techniques (Barr et al., 1995) could be used. Also, linear or planar arrays of sonic anemometers could be employed, such as in Tong et al. (1997). This is likely to provide valuable information for the development of improved SGS model formulations.

\section{Acknowledgements}

The authors gratefully acknowledge the field assistance of Anthony Cahill, Jozsef Szilagy, Teresa Ortenburger, and Mike Mata and the support from the UC Davis Superfund Project 5P42ES04699-07, NSF Grant CTS-9408344, and the Department of Geography and Environmental Engineering at the Johns Hopkins University. The first author received partial support from the "La Caixa" Fellowship Program (Barcelona, Spain).

\section{References}

Akselvoll, K. and Moin, P.: 1996, 'Large-Eddy Simulation of Turbulent Confined Coannular Jets', J. Fluid Mech. 315, 387-411. 
Albertson, J. D., Parlange, M. B., Kiely G., and Eichinger, W. E.: 1997, 'The Average Dissipation Rate of Turbulent Kinetic Energy in the Neutral and Unstable Atmospheric Surface Layer', $J$. Geophysical Res. 102(D12), 13423-13432.

Albertson, J. D. and Parlange, M. B.: 1997, 'Surface Length Scales and Shear Stress: Implications for Land-Atmosphere Interaction over Complex Terrain', Water Resour. Res., submitted.

Andren, A., Brown, A. R., Graf, J., Mason, P. J., Moeng C.-H., Nieuwstadt, F. T. M., and Schumann, U.: 1994, 'Large-Eddy Simulation of the Neutrally Stratified Boundary Layer: A Comparison of Four Computer Codes', Quart. J. Roy. Meteorol. Soc. 120, 1457-1484.

Barr, S., Buttler, W., Clark, D., Cottingame, W., and Eichinger, W.: 1994, 'Lidar-Observed Wind Patterns in the New Mexico-Texas Border Region, 9-11 September, 1994', Report to the ATR, $37 \mathrm{pp}$.

Clark, R. G., Ferziger, J. H., and Reynolds, W. C.: 1979, 'Evaluation of Subgrid Models using an Accurately Simulated Turbulent Flow', J. Fluid Mech. 91, 1-16.

Deardorff, J. W.: 1970, 'A Numerical Study of Three-Dimensional Turbulent Channel Flow at Large Reynolds Numbers', J. Fluid Mech. 41, 453-480.

Deardorff, J. W.: 1974, 'Three-Dimensional Numerical Study of the Height and Mean Structure of a Heated Planetary Boundary Layer', Boundary-Layer Meteorol. 7, 81-106.

Domaradski, J. A., Liu, W., and Brachet, M. E.: 1993, 'An Analysis of Subgrid-Scale Interactions in Numerically Simulated Isotropic Turbulence', Phys. Fluids A 5, 1747-1759.

Dwyer, M. J., Patton, E. G., and Shaw, R. H.: 1997, 'Turbulent Kinetic Energy Budgets from a LargeEddy Simulation of Airflow above and within a Forest Canopy', Boundary-Layer Meteorol. 84, $23-43$.

Gao, W., Shaw, R. H., and Paw U, K. T.: 1989, 'Observation of Organized Structure in Turbulent Flow within and above a Forest Canopy', Boundary-Layer Meteorol. 47, 349-377.

Gao, W. and Shaw, R. H.: 1992, 'Conditional Analysis of Temperature and Humidity Microfronts and Ejection/Sweep Motions within and above a Deciduous Forest', Boundary-Layer Meteorol. 59, 35-57.

Germano, M., Piomelli, U., Moin, P. and Cabot, W.: 1991, 'A Dynamic Subgrid-Scale Eddy Viscosity Model', Phys. Fluids A 3, 1760-1765.

Ghosal, S., Lund, T. S., Moin. P., and Akselvoll, K.: 1995, 'A Dynamic Localization Model for Large-Eddy Simulation of Turbulent Flows', J. Fluid Mech. 286, 229-255.

Härtel, C. and Kleiser, L.: 1993, 'Energy Transfer between Large and Small Scales in Wall-Bounded Turbulent Flows', in U. Piomelli and S. Ragab (eds.), Engineering Applications of Large Eddy Simulations, Vol. 162, ASME., FED, p. 21.

Katul, G., Kuhn, G., Schieldge, J., and Hsieh C.-I.: 1997a, 'The Ejection-Sweep Character of Scalar Fluxes in the Unstable Surface Layer', Boundary-Layer Meteorol. 83, 1-26.

Katul, G., Hsieh, C.-I., and Sigmon, J.: 1997b, 'Energy-Inertial Scale Interactions for Velocity and Temperature in the Unstable Atmospheric Surface Layer', Boundary-Layer Meteorol. 82, 49-80.

Kiely, G., Albertson, J. D., Parlange, M. B., and Eichinger, W. E.: 1996, 'Convective Scaling of the Average Dissipation Rate of Temperature Variance in the Atmospheric Surface Layer', Boundary-Layer Meteorol. 77, 267-284.

Lilly, D. K.: 1967, 'The Representation of Small-Scale Turbulence in Numerical Simulation Experiments', in Proc. IBM Scientific Computing Symposium on Environmental Sciences, 195-210.

Liu, S., Meneveau, C., and Katz, J.: 1994, 'On the Properties of Similarity Subgrid-Scale Models as Deduced from Measurements in a Turbulent Jet', J. Fluid Mech. 275, 83-119.

Mason, P. J. and Thomson, D. J.: 1992, 'Stochastic Backscatter in Large-Eddy Simulations of Boundary Layers', J. Fluid Mech. 242, 51-78.

Mason, P.: 1994, 'Large-Eddy Simulation: A Critical Review of the Technique', Quart. J. Roy. Meteorol. Soc. 120, 1-26.

Meneveau, C.: 1994, 'Statistics of Turbulence Subgrid-Scale Stresses: Necessary Conditions and Experimental Tests', Phys. Fluids 6, 815-833. 
Monin, A. and Yaglom, A.: 1971, Statistical Fluid Mechanics, MIT Press, Cambridge MA, 874 pp.

Murray, J. A., Piomelli, U., and Wallace, J. M.: 1996, 'Spatial and Temporal Filtering of Experimental Data for a priori Studies of Subgrid-Scale Stresses', Phys. Fluids 8, 1978-1980.

Nieuwstadt, F. T. M., Mason P. J., Moeng, C.-H., and Schumann, U.: 1991, 'Large-Eddy Simulation of the Convective Boundary Layer: A Comparison of Four Computer Codes', Turbulent Shear Flows 8, 343-367.

O'Neil, J. and Meneveau, C.: 1997, 'Subgrid-Scale stresses and their Modeling in a Turbulent Plane Wake', J. Fluid Mech. 349, 253-293.

Parlange, M. B., Katul, G. G., Folegatti, M. V., and Nielsen, D. R.: 1993, 'Evaporation and the Field Scale Soil Water Diffusivity Function', Water Resour. Res. 29, 1279-1286.

Peltier, L. J., Wyngaard, J. C., Khanna, S., and Brasseur, J. G.: 1996, 'Spectra in the Unstable Surface Layer', J. Atmos. Sci. 53, 49-61.

Piomelli, U., Moin, P., and Ferziger, J. H.: 1988, 'Model Consistency in Large Eddy Simulation of Turbulent Channel Flows', Phys. Fluids 31, 1884-1891.

Porté-Agel, F., Meneveau, C., and Parlange, M. B.: 1998, 'A Scale-Dependent Dynamic Model for Large-Eddy Simulation: Application to the Atmospheric Boundary Layer', submitted.

Press, W. C., Teukolsky, S. A., Vetterling, W. T., and Flannery, B. P.: 1992, Numerical Recipes, Cambridge University Press, 2nd edition, 963 pp.

Raupach, M. R., Antonia, R. A., and Rajagopalan, S.: 1991, 'Rough-Wall Turbulent Boundary Layers', Appl. Mech. Rev. 44, 1-25.

Schumann, U.: 1995, 'Stochastic Backscatter of Turbulence Energy and Scalar Variance by Random Subgrid-Scale Fluxes', Proc. R. Soc. Lond. A 451, 293-318.

Shaw, R. H., Tavangar, J., and Ward, D.: 1983, 'Structure of the Reynolds Stress in a Canopy Layer', J. Clim. Appl. Meteorol. 22, 1922-1931.

Shaw, R. H., Paw U, K. T., and Gao, W.: 1989, 'Detection of Temperature Ramps and Flow Structures at a Deciduous Forest Site', Agric. For. Meteorol. 47, 123-138.

Shaw, R. H. and Schumann, U.: 1992, 'Large-Eddy Simulation of Turbulent Flow above and within a Forest', Boundary-Layer Meteorol. 61, 47-64.

Smagorinsky, J.: 1963, 'General Circulation Experiments with the Primitive Equations, Part 1: The Basic Experiment', Monthly Wea. Rev. 91, 99-164.

Sorbjan, Z.: 1996, 'Effects Caused by Varying strength of the capping inversion based on a large-eddy simulation of the shear-Free Convective Boundary Layer', J. Atmos. Sci. 53, 2015-2024.

Tong, C., Wyngaard, J. C., Khanna, S., and Brasseur, J. G.: 1997, 'Resolvable- and Subgrid-Scale Measurement in the Atmospheric Surface Layer: Technique and Issues', J. Atmos. Sci., preprint.

Wyngaard, J. C. and Clifford, S. F.: 1977, 'Taylor's Hypothesis and High-Frequency Turbulence Spectra', J. Atmos. Sci. 34, 922-929. 